\title{
Linx
}

Revue des linguistes de l'université Paris X Nanterre

47 | 2002

Du sens au sens

\section{La nuit, certains chats sont gris, ou la généricité sans syntagme générique}

Some cats are grey in the dark

Jean-Claude Anscombre

\section{(2) OpenEdition}

Journals

Édition électronique

URL : http://journals.openedition.org/linx/558

DOI : $10.4000 /$ linx.558

ISSN : 2118-9692

Éditeur

Presses universitaires de Paris Nanterre

Édition imprimée

Date de publication : 1 décembre 2002

Pagination : 13-30

ISSN : 0246-8743

\section{Référence électronique}

Jean-Claude Anscombre, «La nuit, certains chats sont gris, ou la généricité sans syntagme

générique », Linx [En ligne], 47 | 2002, mis en ligne le 01 juin 2003, consulté le 20 avril 2019. URL:

http://journals.openedition.org/linx/558; DOI : 10.4000/linx.558

Département de Sciences du langage, Université Paris Ouest 


\title{
La nuit, certains chats sont gris, ou la généricité sans syntagme générique
}

\author{
Jean-Claude Anscombre, C.N.R.S. (UMR 7546), E.H.E.S.S. \\ (CELITH)
}

\section{Avant-propos}

Les notions de généricité, de phrase générique et de syntagme générique n’ont pas bonne réputation en linguistique. On les voit généralement comme la manifestation d'une passion exagérée pour la formalisation et la « logique » des langues naturelles, et donc comme un phénomène marginal. Il est vrai que, bien souvent, leur étude s'est bornée aux phrases analytiques stricto sensu' ${ }^{1}$, et qui plus est, à sujet générique. Ce qui permettait en outre de rapprocher l'article défini qui y figurait du quantificateur universel $\forall$. Bien peu de linguistes, à ma connaissance, se sont penchés sur la place effective qu'occupent la généricité et les phrases génériques dans l'édifice langagier.

La première section du présent travail examine brièvement les propriétés basiques des phrases génériques de bon aloi, et reprend une distinction classique entre différents types de généricité. La seconde section examine un cas de phrase générique non répertorié, lequel met en évidence la non nécessité d’un syntagme générique en position sujet. Dans la troisième section enfin, j’aborderai le rôle que jouent les phrases génériques en morpho-sémantique.

La plupart des problèmes étudiés ici nécessiteraient clairement des développements plus importants. Mon seul but est d'attirer l'attention sur des phénomènes qui me paraissent non marginaux comme on l'a longtemps cru, mais centraux en sémantique.

\section{Les phrases génériques : le point de vue traditionnel}

\subsection{Les différents types de généricité}

Les énoncés suivants :

(1) Les chats chassent les souris.

${ }^{1}$ Il est vrai que les phrases analytiques jouent un rôle capital en sciences, et plus généralement dans la connaissance et la philosophie de la connaissance. 
Jean-Claude Anscombre
(2) Les éléphants sont rancuniers.
(3) Les chimpanqés sont des singes.
(4) Aucun célibataire n'est marié.
(5) Les baleines sont des mammiferes
(6) Les enfants aiment les bonbons.
(7) Les chats sont affectueux.
(8) Les singes mangent des bananes.

«classiques » de la «littérature »sur la généricité, illustrent de façon exemplaire la conception traditionnelle : est générique une phrase qui : a) Est vraie (en un sens qui reste à préciser); b) N'est pas événementielle; c) Possède un syntagme sujet générique, habituellement de la forme les $N^{2}$. Les points a) et b) sont des lieux communs des études sur la généricité, et tous les auteurs ayant abordé ce sujet - ainsi [Carlson, 1982], [Dahl, 1985], [Galmiche, 1985], [Kleiber, 1978], [Kuroda, 1973],...etc., ont insisté sur le fait que les phrases génériques ne concernent pas des événements spécifiques, mais des états de choses généraux et habituels. Les phrases génériques ne sont pas événementielles, mais dénotent des vérités générales ${ }^{3}$ : elles sont gnomiques. Pour ce qui est de c) - le sujet grammatical est un syntagme générique $^{4}$, c'est une des vulgates les plus diffusées, par exemple [Carlson, 1982], [Jackendoff, 1985], [Jespersen, 1971], [Mc Cawley, 1981],... etc. Ont pu contribuer à cet état de choses une certaine tradition syllogistique, ainsi que l'analyse des déterminants en termes de quantificateurs logiques. Quoi qu'il en soit, sera pour l'instant générique toute phrase possédant les traits a), b), et c). Je vais maintenant mettre en évidence les propriétés linguistiques de ces phrases génériques. Sur la base de ces propriétés, je proposerai une classification. Je montrerai ensuite que certaines phrases ne possédant que les traits a) et b), mais non c) manifestent cependant des propriétés analogues, et qu'on doit donc aussi les considérer comme génériques.

Je me bornerai dorénavant à l'étude des phrases génériques vraies (ou présentées comme telles). Je rappelle qu'on distingue habituellement, parmi les phrases vraies ou valides ${ }^{5}$, les phrases vraies a priori, et les phrases synthétiques. Est phrase vraie a priori tout phrase dont la valeur de vérité est indépendante de toute vérification empirique, et phrase synthétique celle dont la valeur de vérité n'est

2 On trouve également citées, bien que moins fréquemment, des phrases génériques en $L e N$ et en Un N, par exemple : Le lynx a une vue perçante, L'or est un métal précieux, Une taupe déblaie une tonne de terre par jour, Un diplôme médiocre vaut mieux que pas de diplôme,...etc. Les trois formes n'existent pas toujours concurremment. Par ailleurs, il est très rare de rencontrer des phrases génériques au passif, ainsi : Les civilisations méditerranéennes sont étudiées par mon fils, qui, lorsqu'elle est possible, ne semble pas être sémantiquement équivalente à Mon fils étudie les civilisations méditerranéennes. Enfin, la généricité de phrases comme Des diplomates doivent être discrets n'a guère fait l'objet d'études.

${ }^{3}$ Bien que l'on puisse étudier, et cela a été fait, des phrases génériques fausses, comme Les voitures ont des roues carrées.

${ }^{4}$ Il ne semble pas que personne se soit occupé de la possible généricité de phrases comme Max déteste les éclairs au chocolat ou encore Marie est coquette avec les hommes, à syntagme générique non sujet. Le découpage thème/propos n'est probablement pas étranger à ce phénomène.

5 Pour une synthèse documentée sur le sujet, cf. [Kleiber, 1978]. 
déterminable que par comparaison avec l'empiriquement vrai. Des deux énoncés Les chats ont quatre pattes et Colomb découvrit l'Amérique en 1492, seul le premier est a priori, le second étant synthétique. Les phrases vraies a priori se divisent à leur tour en phrases nécessairement vraies, généralement vraies et vraies par convention. Voici un exemple de chaque type respectivement :

$$
\begin{aligned}
& \text { (9) Les autruches sont des oiseaux. } \\
& \text { (10) Les avions ont des ailes. } \\
& \text { (11) } 2579 \text { est un nombre premier. }
\end{aligned}
$$

Les phrases comme (9) et (10) sont valides en vertu de la seule signification des mots qui les composent: ce sont respectivement les phrases analytiques et les phrases typifiantes a priori ${ }^{6}$. Pour ce qui est de (11), sa valeur de vérité se fonde non sur une compétence linguistique, comme c'est le cas pour (9) et (10), mais sur une compétence mathématique, i.e. sur des conventions ${ }^{7}$. Je laisserai de côté ici les phrases vraies par convention, et me bornerai de plus aux phrases génériques. Ce qui nous donne la classe des phrases génériques vraies a priori, laquelle se subdivise en phrases génériques nécessairement vraies (les analytiques, type A), les génériques généralement vraies (les typifiantes a priori, type B) et les génériques synthétiques (les typifiantes locales, type $\mathrm{C})^{8}$. Voici un exemple de chaque type :

(3) Les chimpanzés sont des singes (type A).

(8) Les singes mangent des bananes (type B).

(12) Les singes sont amusants (type C).

Rappelons brièvement quelques unes des propriétés linguistiques les plus connues permettant de différencier ces trois types.

\subsection{Quelques propriétés des phrases génériques}

Voici quelques-unes des différences les plus saillantes entre ces trois types de phrases génériques?

\footnotetext{
${ }^{6}$ Pour ne pas compliquer un sujet déjà fort complexe, je ne suivrai pas ceux qui appellent analytiques aussi bien les phrases comme (9) que celles comme (10), bien qu'elles n'aient pas les mêmes propriétés. Enfin, je n'entrerai pas dans le débat sur le bien-fondé philosophique de la distinction analytique/synthétique. On pourra se reporter à [Martin, 1985], [Putnam, 1975], [Quine, 1963], [Quinton, 1967],... etc.

${ }^{7} \mathrm{La}$ limite entre les vérités analytiques et les vérités par convention n'en reste pas moins floue. Ainsi Deux est pair ou La terre tourne autour du soleil peuvent être vues comme analytiques ou vraies par convention selon que l'on considère qu'elles font partie du langage de tous les jours ou d'une langue technique. C'est le problème général des vérités scientifiques quand elles tombent dans le domaine commun.

${ }^{8}$ Cf. sur tous ces points, [Anscombre, 1995, 1995-96, 1996, 1999], [Kleiber, 1978, 1987, 1988]).

${ }^{9}$ Je laisse de côté la distinction entre phrase et énoncé, tout en sachant qu'il n’y a généralement pas de phrases génériques dans nos langues, mais des interprétations génériques de certains énoncés. Phénomène dû au fait que nos langues, pour la plupart, ne possèdent pas de marque(s) spécifique(s) de la généricité.
} 
$\left(\mathbf{G}_{\mathbf{1}}\right)$ Déduction syllogistique et raisonnement in absentia : seul le type A permet des syllogismes logiquement valides. Les types $\mathrm{B}$ et $\mathrm{C}$ ne permettent que des raisonnements valides in absentia.

En effet, alors que le syllogisme suivant est impeccable :
(13) 1. Les chimpanzés sont des singes.
2. Cheetab est un chimpanzé.
3. (Donc) Cheetah est un singe.

le raisonnement suivant semble moins assuré :
1. Les singes sont amusants.
2. Cheetah est un singe.
3. (Donc) Cheetab est amusante.

La différence est en fait une différence de nature. Alors que (13) est une authentique déduction, (14) n'est qu'un raisonnement conclusif, et qui parât forcé si on tient à y voir une déduction. Ce que dit (14), c'est que les singes sont amusants, et que si aucune contre-indication factuelle ou situationnelle ne s'y oppose, il est probable que le singe Cheetah est amusant. Une conclusion plus acceptable serait, dans le cas de (14), quelque chose comme Il est vraisemblable que Cheetab est amusante. L'origine de cette différence apparaît clairement au vu de la propriété suivante :

$\left(\mathbf{G}_{2}\right)$ Les phrases génériques de type $\mathrm{B}$ ou $\mathrm{C}$ admettent des exceptions sans perdre leur généricité, les phrases génériques de type A n'admettent aucune exception.

On le vérifie sans peine sur les exemples suivants :

(15) * Cheetah est un chimpanzé, mais ce n'est pas un singe.

(16) Cheetah est un singe, mais elle ne mange pas de bananes.

(17) Cheetah est un singe, mais elle n'est pas amusante.

qui renvoient respectivement à (3), (8), et (12).

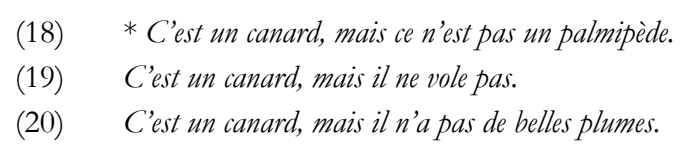

exemples qui renvoient cette fois à Les canards sont des palmipèdes (analytique), Les canards volent (typifiante a priori), Les canards ont de belles plumes (typifiante locale).

On voit ainsi que, contrairement aux phrases analytiques (le type A), les phrases typifiantes (types B et C) présentent une généralité comme n’étant que probable, que de l'ordre du vraisemblable, et ne pouvant donc présenter que des conclusions valables in absentia, i.e. uniquement probables. Les phrases analytiques possèdent la stricte rationnalité du discours scientifique, alors que les phrases typifiantes ne permettent que le raisonnement discursif. Un discours fondé sur des phrases analytiques est logique, et non argumentatif. A l'inverse, un discours fondé sur des phrases typifiantes est argumentatif, mais ne peut être logique, puisqu'il laisse la porte ouverte à de possibles exceptions. 
( $\left.\mathbf{G}_{3}\right)$ Les phrases génériques de type $\mathrm{B}$ et $\mathrm{C}$ se combinent généralement (!) avec des expressions adverbiales comme généralement, normalement, habituellement, en général, d'une façon générale. Cette combinaison est refusée par les phrases génériques du type A.

(21) * (Généralement + habituellement + en général), les chimpanzés sont des singes.

(22) (Généralement + normalement + d'une façon générale), les voitures ont quatre roues.

(23) (Généralement + habituellement + d'une façon générale), les linguistes sont ennuyeux.

On peut remarquer que ce critère semble être une conséquence prévisible du précédent, dans la mesure où les types $\mathrm{B}$ et $\mathrm{C}$ expriment une tendance simplement majoritaire d'une classe d'entités, alors que le type A présente une stricte quantification universelle, incompatible avec la vision sommairement globaliste des deux autres types. Nous y reviendrons plus loin.

$\left(\mathbf{G}_{4}\right)$ La possibilité de SN spécifiques: seules les phrases typifiantes locales admettent les $\mathrm{SN}$ spécifiques, les types A et B ne les admettant pas.

Je rappelle qu'une phrase générique du type Les $S N$ sont $P^{10}$ admet des $\mathrm{SN}$ spécifiques si un locuteur admettant cette généricité trouve sémantiquement acceptable la phrase Ce SN est P. La signification exacte de cette curieuse propriété apparaitra plus loin . Dans le cas des phrases analytiques, elle signifie que dans une communauté linguistique pour laquelle Les cbimpanzés sont des singes est générique, on trouvera bizarre d'asserter Ce chimpanzée est un singe. Plaçons-nous maintenant que dans notre monde occidental actuel, où Les voitures ont quatre roues est générique. Il s'agit alors d'une typifiante a priori (elle admet des exceptions). Supposons que j'apprenne à mon ami Georges que je viens d'acheter une voiture neuve, et qu'il me demande comment est cette voiture : je ne pourrai lui dire - sauf volonté d'ironie ou de moquerie - Cette voiture a quatre rones ${ }^{11}$. Mais un $\mathrm{SN}$ spécifique sera tout à fait possible s'il s'agit de manifester une anormalité par rapport à la phrase générique correspondante, ainsi Cette voiture a six roues, c'est une tout-terrain. En revanche, dans le cas d'une phrase typifiante locale, par exemple Les éléphants sont rancuniers, rien n'empêche d'affirmer que Cet éléphant est rancunier, par exemple de la façon suivante : Les éléphants sont rancuniers, et celui-ci n'échappe pas à la tradition, il est rancunier.

\section{Généricité et syntagme générique}

\section{Un cas de généricité non standard}

Le paragraphe précédent, partant de phrases considérées comme sans conteste génériques, et devant apparemment leur généricité à un syntagme générique (sujet en l'occurrence), établissait les propriétés des trois types de phrases génériques envisagés

\footnotetext{
${ }^{10}$ La graphie Les $S N$ sont $P$ symbolise une phrase générique affirmant qu'un $\mathrm{SN}$ a la propriété $\mathrm{P}$, même si cette assertion ne se fait pas par le biais de la copule.

${ }^{11}$ Une telle phrase est en revanche possible comme non réponse: Cette voiture a quatre roues, comme toutes les voitures!
} 
dans cette étude. Je procèderai maintenant à l'inverse: je montrerai que certaines phrases en certains possèdent les propriétés que nous avons dégagées, et doivent donc être considérées comme étant elles aussi génériques. Je partirai d'exemples comme :

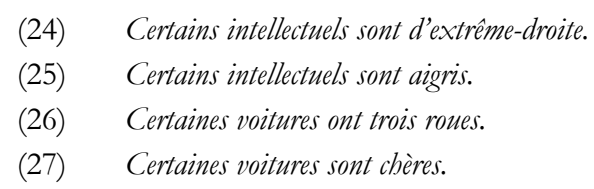

Notons tout d'abord que si la phrase de base Les $x$ sont $P$ est générique, Certains $x$ sont $P$ n'est pas assertable. En d'autres termes, Certains chimpanzés sont des singes n'est pas une phrase sémantiquement bien formée. Plus, ce phénomène n'est pas dù à l'analycité de la phrase considérée. Toute personne considérant comme valide Les voitures ont quatre roues, ne peut cependant asserter Les voitures - enfin certaines voitures - ont quatre roues ${ }^{12}$ et conserver la généricité de Les voitures ont quatre roues. Or les phrases du type de (24) à (27) satisfont en fait à beaucoup des critères de la généricité. On notera qu'elles se présentent comme vraies, et qu'elles permettent au moins une interprétation non événementielle : elles représentent une propriété. Il y a plus : de

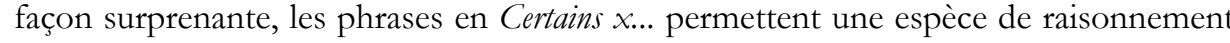
in absentia (critère $\mathbf{G}_{\mathbf{1}}$ ). Supposons qu'on me demande mon avis sur Max, l'intellectuel bien connu. L'enchaînement ci-après constitue sans surprise une réponse tout à fait valide, reposant en fait sur un tel raisonnement in absentia:

(28) Max est certainement aigri, certains intellectuels le sont.

Ou encore, on me somme de caractériser la voiture révolutionnaire du prochain salon, à propos de laquelle je pourrai formuler, de façon analogue, le jugement suivant :

(29) Elle a peut-être trois rones, certaines voitures ont trois rones.

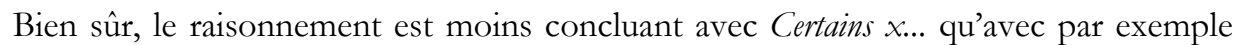
Tous les $x . .$. Rien d'étonnant à cela : ce que l'on appelle raisonnement in absentia n'est tout bien considéré qu'une argumentation. Et dans ce domaine, Tous les x... est un argument supérieur à Certains x... comme le montre l'enchaînement par même :

(30) Certains intellectuels, et même tous les intellectuels, sont aigris.

On aura noté au passage une surprenante propriété de la langue. Dire d'une classe C d'entités, au moyen d'une phrase telle que celles qui viennent d'être vues, que certaines d'entre elles ont la propriété $\mathrm{P}$, revient d'une certaine façon à qualifier la classe $\mathrm{C}$ tout entière. En effet, la généricité de la phrase en Certains... fait de l'appartenance à $\mathrm{C}$ un argument plausible pour la possession de $\mathrm{P}$, et la teinte en quelque sorte de P-itude. C'est ce qui fait qu'une phrase comme Certains chiens attaquent l'homme, qui ne concerne en apparence qu'une sous-classe des chiens, peut cependant être avancée pour justifier une méfiance étendue à l'intégralité de la classe : Méfie-toi des chiens: certains attaquent l'homme. Passons maintenant au problème des éventuelles exceptions, i.e. à l'examen du critère $\left(\mathbf{G}_{2}\right)$. Remarquons que le problème n'est pas de

${ }^{12}$ Cet énoncé est tout à fait possible comme rectification de la phrase générique. 
montrer que dans la classe des entités $\mathrm{x}$ dont on prédit que Certains $x$ sont $P$, il y a des $\mathrm{x}$ qui ne sont pas $\mathrm{P}$, car il s'agit là d'un pur truisme. Ce qu'il faut en fait montrer, c'est que, dans le cas où ces certains $\mathrm{x}$ qui sont $\mathrm{P}$ renvoient à une classe par ailleurs identifiable, l'existence d'éventuelles exceptions n'invalide pas la phrase en Certains...Il en est ainsi en particulier chaque fois qu’à ces certains est associée une dénomination. Supposons en effet que les $\mathrm{x}$ qui sont $\mathrm{P}$ soient habituellement dénommés des $y$. A la phrase Certains $x$ sont $P$ est par conséquent associée la phrase cette fois à syntagme générique Les y sont $P$. On est alors en droit de s'attendre à une étroite parenté entre Certains $x$ sont $\mathrm{P}$ et Les $y$ sont $\mathrm{P}$ pour ce qui est des propriétés linguistiques. En voici un premier exemple. Considérons la phrase :

(31) Certains entiers n'ont pas d'autres diviseurs qu'eux-mêmes et l'unité.

De tels entiers sont bien connus : ce sont les fameux nombres premiers. A cette phrase correspond donc la phrase générique «standard » :

(32) Les entiers premiers n'ont pas d'autres diviseurs qu'eux-mêmes et l'unité.

Or (32) est analytique, et n'admet pas d'exceptions: on est alors conduit à voir également dans (31) une phrase analytique, et qui comme telle, n'admet pas d'exceptions. On ne peut pas être un entier premier et admettre d'autres diviseurs que soi-même et l'unité. Passons maintenant à des caractérisations qui induisent des phrases non pas analytiques, mais simplement typifiantes, que ce soit localement ou a priori. Considérons à ce titre :

(33) Certaines voitures de cette série ont la direction assistée.

(34) Certaines voitures de cette gamme consomment beaucoup.

phrases auxquelles correspondent les versions génériques « standard» :

(35) Les voitures $X$ de cette série ont la direction assistée.

(36) Les voitures $Y$ de cette gamme consomment beaucoup.

respectivement typifiante a priori et locale, avec possibilités d'exceptions :

(37) Les voitures $X$ de cette série ont la direction assistée, sauf celles achetées à crédit.

(38) Les voitures $Y$ de cette gamme consomment beaucoup, sauf si elles marchent au fuel.

Or ce qui est tout à fait remarquable en l'occurrence, c'est que ces mêmes éventuelles exceptions sont possibles avec les versions en certains, comme on peut le constater sur :

(39) Certaines voitures de cette série ont la direction assistée, sauf celles achetées à crédit.

(40) Certaines voitures de cette gamme consomment beaucoup, sauf si elles marchent au fuel.

En revanche, les phrases en Certains X...ne satisfont pas le critère $\left(\mathbf{G}_{3}\right)$ :

(41) ?? (Généralement + habituellement), certains enfants aiment les bonbons.

(42) ?? (Normalement + d'une façon générale), certains mensonges ont leur utilité.

(43) $\quad\left({ }^{*}\right.$ Généralement $+*$ d'une façon générale + ??normalement), certains ouvrages sont reliés plein cuir. 
Examinons ce phénomène pour tenter d'en rendre compte. Notons que l'explication qui y verrait une incompatibilité entre généralement qui ne renverrait qu'à un phénomène général sur une classe, et certains qui ne mettrait en jeu qu'une propriété d'une partie de cette même classe, est par trop simpliste. On peut en effet remarquer que tous ne se combine pas non plus avec généralement, bien qu'il dise d'un phénomène qu'il est général sur toute une classe :

\section{(44) ?? Généralement, tous les enfants aiment les bonbons.}

Pour résoudre ce problème, je ferai un détour par les phrases habituelles et leurs propriétés, étudiées entre autres par Carlson; 1982, Guericolas; 1987 et Kleiber ; 1987. Étudiant les différences entre des phrases comme :

(45) Max va au lycée (habituelle dispositionnelle).

(46) Max va au lycée à pied (habituelle fréquentative).

ces auteurs remarquent : a) que les habituelles dispositionnelles comme (45) admettent une pseudo-paraphrase par une structure attributive nominale, mais non les habituelles fréquentatives comme (46):

$$
\begin{aligned}
& \text { (45a) Max est lycéen. } \\
& \text { (46a)* Max est lycéen à pied. }
\end{aligned}
$$

Et: b) que les habituelles fréquentatives admettent une paraphrase en Quand $p, q$ impossible avec les habituelles dispositionnelles :

$$
\begin{aligned}
& \text { (45b) * Quand Max va, c'est au lycée. } \\
& \text { (46b) Quand Max va au lycée, il le fait à pied. }
\end{aligned}
$$

Or, fait extrêmement intéressant, seules les habituelles fréquentatives admettent la combinaison avec des adverbes comme généralement, habituellement, normalement:

$$
\begin{aligned}
& (45 \mathrm{c}) * \text { (Généralement }+ \text { habituellement }+ \text { normalement), Max va au lycée }{ }^{13} . \\
& \text { (46c) (Généralement }+ \text { habituellement }+ \text { normalement), Max va au lycée à pied. }
\end{aligned}
$$

Je vais m'inspirer de ce qui précède pour traiter le problème qui nous occupe. A cet effet, je considèrerai les trois phrases ${ }^{14}$ :
(47) Les castors construisent des barrages.
(48) Certains castors mangent des carottes.
(49) Certains castors construisent des barrages en pierre.

La combinaison avec généralement - la seule que j'examinerai ici ${ }^{15}$ - conduit aux observation suivantes :

13 De telles combinaisons sont en fait possibles, mais moyennant une importante modification du sens de (45).

14 (48) et (49) sont visiblement inventées de toutes pièces, en fait pour des raisons pédagogiques.

15 Normalement, babituellement, et généralement sont souvent classés dans la même catégorie d'adverbes : adverbes-cadre pour S. Schlyter (La place des adverbes en -ment en français, Thèse, 1977, Constance), et 
(47a) Généralement, les castors construisent des barrages.

(47b) Les castors construisent généralement des barrages.

(48a)* Généralement, certains castors mangent des carottes.

(48b) ?? Certains castors mangent généralement des carottes.

(49a) * Généralement, certains castors construisent des barrages en pierre.

(49b) Certains castors construisent des barrages généralement en pierre.

Ces exemples conduisent à deux remarques : d'une part, et assez visiblement pour des questions de portée, la combinaison de (47), (48), et (49), avec généralement dépend de la place de cet adverbe dans la phrase. D'autre part, ces trois phrases admettent la paraphrase par une structure attributive nominale :

(47c) Les castors sont des constructeurs de barrages.

(48c) Certains castors sont des mangeurs de carottes.

(49c) Certains castors sont des constructeurs de barrages en pierre.

Ce qui tendrait à les rapprocher des habituelles dispositionnelles. Il est en fait erroné de considérer que la combinaison avec généralement a quelque chose à voir avec le caractère dispositionnel. En effet, selon les critères énoncés plus haut, Max aime le chocolat est une phrase dispositionnelle, non combinable avec généralement. Mais une phrase comme Les enfants aiment le chocolat est tout aussi dispositionnelle que la précédente, et se combine cependant avec l'adverbe en question. En fait, généralement n'est possible qu'avec des structures paraphrasables en si $p$, $q-$ le si pouvant être temporel dans le cas par exemple des habituelles fréquentatives. L'adverbe commente alors le caractère générique du lien entre $p$ et $q$ : si on a $p$, c'est généralement le cas qu'on ait q. (47) admet une telle paraphrase: "Si x est un castor, x construit des barrages », mais non (48), on le constate immédiatement. En revanche, on a pour (49) : «Si certains castors construisent des barrages, ceux-ci sont en pierre ». D'où les phénomènes signalés : (47) se combinera avec l'adverbe, de même que (49), mais non (48). Pour ce qui est de la place, la règle semble être que généralement peut «monter» jusqu'à occuper la place qui précède directement le segment quantifié sur lequel il porte. D'où la convergence entre (47a) et (47b), et la divergence entre (49a) et (49b). On voit ainsi que le critère de la combinabilité avec généralement n'est pas un critère simple de généricité. Tout ce que permet de dire ce critère, c'est que sa satisfaction ne peut être due à la présence d'un syntagme en certains.

Naturellement, on est amené à se poser la question du type de généricité des phrases en Certains x... Nous avons vu qu'elles peuvent être analytiques. Lorsqu'elles ne le sont pas, il reste à déterminer si elles appartiennent au type B (typifiantes a priori) ou du type $C$ (typifiantes locales). C'est ainsi qu'on peut penser à appliquer le critère $\left(\mathbf{G}_{4}\right)$ relatifs aux $\mathrm{SN}$ spécifiques. On obtient :

(50) Certains enfants aiment les bonbons.

(51) Cet enfant aime les bonbons.

adverbes d'habitude (sous-classe des adverbes disjonctifs) pour C. Molinier, «Une classification des adverbes en -ment $»$, Langue française, 1990, 88, pp. 28-40. 
Jean-Claude Anscombre

Certains livres sont luxueusement reliés.

Ce livre est luxueusement relié.

Ce qui semblerait prouver que les phrases en Certains $x$ sont $P$ sont des génériques typifiantes locales. Cette conclusion (provisoire) ne laisse pas de surprendre, et invite à examiner le phénomène de plus près. Soit en effet les deux phrases :

(54) Certains Français sont blonds.

(55) Certaines voitures ont six roues.

Or [Kleiber, 1978] observe qu'on ne peut interroger que les phrases génériques typifiantes locales, jamais les a priori, qu'elles soient d'ailleurs analytiques ou seulement typifiantes. Ce qu'illustrent les exemples ci-après :

(56) * Est-ce que les chimpanzés sont des singes?

(57) * Est-ce que les castors construisent des barrages?

(58) Est-ce que les linguistes sont sympathiques?

Appliquons ce critère à nos exemples, on obtient le curieux résultat suivant :

(59) * Est-ce que certains français sont blonds?

(60) Est-ce que certaines voitures ont six roues?

C'est qu'en fait (54) et (55) présentent une différence fondamentale qui n'est pas immédiatement perceptible. Elle réside en ce que connaître le sens du mot voiture c'est admettre l'existence d'une phrase générique valide Les voitures ont quatre roues attachée à ce terme, phrase typifiante a priori. Une telle phrase est susceptible d'admettre des exceptions : c'est précisément à de telles exceptions que renvoie Certaines voitures ont six roues. Rien de tel en revanche dans le cas de (54). Car connaître le sens du mot Français, c'est être capable d'attacher à ce terme des phrases comme Certains Français sont blonds, bruns, roux,...etc. Mais il n'existe pas, comme pour (54), de phrase en Les Français sont $C$, où $\mathrm{C}$ serait une couleur de cheveux stéréotypique du français, couleur par rapport à laquelle être blond constituerait une éventuelle exception. Appelons extensive une phrase générique de type Les $N$ sont $P$, et partitive une phrase générique de type Certains $x$ sont $P$. Les critères habituels distinguant les divers types de généricité montrent donc que dans le cas des phrases partitives comme dans celui des extensives, il y a des partitives analytiques (Certains entiers n'ont pas d'autres diviseurs qu'eux-mêmes et l'unitê) ; des partitives typifiantes a priori, lorsque Certains $x$ sont $P$ s'oppose à d'autres partitives Certains $x$ sont $Q$; et des partitives typifiantes locales, lorsque Certains $x$ sont $P$ dénote une exception par rapport à Les $x$ sont $Q$.

\subsection{De la définition de la généricité}

Au vu de ce qui précède, le concept de généricité n'est pas sans poser quelques problèmes. Il est en effet habituel de décrire les génériques du type Les $x$ sont $P$ comme attribuant à la classe $\mathrm{C}$ des $\mathrm{x}$ la détention de la propriété $\mathrm{P}^{16}$. Dans le cas d'une

\footnotetext{
16 «...Faire une phrase générique, c'est énoncer une propriété qui appartient à un genre donné... », [Galmiche, 1985, p. 14].
} 
analytique, cette analyse ne pose guère de problème. La simple appartenance à $\mathrm{C}$ confère à une entité $\mathrm{x}$ la propriété $\mathrm{P}$, selon le mécanisme logique bien connu. Mais dans le cas d'une typifiante, le problème est plus complexe, puisque l'existence d'éventuelles exceptions oblige à dire de $\mathrm{P}$ qu'elle n'est que majoritaire. Mais on peut encore parler de P comme qualifiant «globalement» la classe C. Que dire alors d'une générique partitive en Certains... ? Quel mécanisme bizarre lui confère une généricité que sa forme superficielle semble démentir d'entrée ? Est-il possible, dans de telles conditions, de donner une définition unique de la généricité ?

Pour répondre à ces différentes questions, je reprendrai une étude déjà ancienne de [Geach, 1967], reprise par [Ducrot, 1970], dans laquelle Geach étudie des énoncés du type de Certains amis sont venus me voir ou Quelques chats sont blancs. Geach y critique la doctrine de la distribution selon laquelle, dans de tels énoncés, les syntagmes nominaux Certains amis, Quelques chats,...etc., réfèrent, i.e. ont pour fonction sémantique de désigner certains éléments de l'univers du discours, de délimiter l'extension d'un concept. En disant Certains amis sont venus me voir, je vous parle de certains de mes amis à propos desquels je prédique qu'ils sont venus me voir, selon une analyse en sujet-prédicat tout à fait parallèle à celle de Pierre est venu me voir. Or cette analyse échoue à rendre compte de phénomènes comme la divergence de comportement entre :

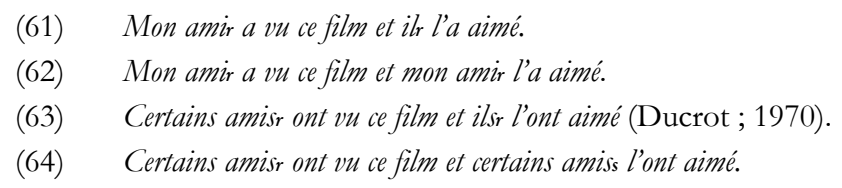

Alors que dans (61) Mon ami et il co-réfèrent, ainsi que Mon ami et mon ami dans (62), (63) et (64) divergent. Certains amis et ils co-réfèrent dans (63), alors que les deux occurrences de Certains amis de (64) ne sont pas co-référentielles. A l'inverse, Geach analyse un énoncé comme Certains amis sont venus me voir comme référant fondamentalement à la classe de mes amis, dont je dis que certains sont venus me voir (ou des amis qui sont venus me voir, dont je prédique que ce sont certains). On peut remarquer que certaines propriétés linguistiques vont dans le sens de Geach. Selon son analyse en effet, les phrases en Certains X... ont la classe des x comme thème, et Certains... comme propos. On constate effectivement que certains - mais également beaucoup - sont possibles dans les positions correspondant à un tel découpage, mais n'aiment guère le découpage inverse :

(65) (Certains + beaucoup) de ces verbes possèdent une propriété caractéristique.

(66) Quant à ces verbes, (certains + beaucoup) possèdent une propriété caractéristique.

(67) * Quant à (certains + beaucoup), ces verbes possèdent une propriété caractéristique.

Enfin, on peut reprendre Certains $x$ par anaphore pronominale, mais aussi $x$ seul, comme illustré sur :

(59) [Certains [éléphants]r]s vivent centenaires, et alors ilsr meurent d'un seul coup.

(60) [Certains [éléphants]r]s vivent centenaires, mais en général, ilss vivent moins longtemps. 
On voit donc, à la lumière de l'analyse de Geach, le mécanisme qui concourt à la généricité des phrases Certains $\times$ sont $P$. De telles phrases attribuent à la classe des $\mathrm{x}$ la propriété [certains sont $\mathrm{P}$ ]. Mais cela seul ne suffit pas à assurer la généricité, si du moins nous voulons caractériser non seulement la classe dans son ensemble, mais aussi chacun de ses éléments. Nous y parviendrons en fait en établissant entre la généricité et le raisonnement conclusif le lien suivant:

(Déf) Une phrase $G$ est générique si :

a) $G$ est présentée comme valide.

b) G n'est pas événementielle, mais est une phrase-propriété (stative).

c) $G$ fait intervenir une classe $C$ d'entités et une propriété $P$ telles que l'appartenance d'une entité $\mathrm{x}$ à la classe $\mathrm{C}$ est un argument pour que $\mathrm{x}$ possède la propriété $\mathrm{P}$.

De ce point de vue, les génériques analytiques sont bien des génériques, mais d'un type particulier : elles ne correspondent en effet pas à une argumentation, mais à une implication. D'après cette définition, les phrases Certains $x$ sont $P$ - mais sans doute également La plupart des $x$ sont $P$, Tous les $x$ sont $P$, Beaucoup de $x$ sont $\mathrm{P}-$ ont autant droit que Les $x$ sont $P$ à l'appellation de "phrase générique ». On en déduit que dans de telles phrases, le syntagme nominal n'est pas à proprement parler un marqueur de généricité, mais un marqueur de type de généricité, ainsi qu'un indicateur de force argumentative. Certaines phrase génériques fournissent un argument plus fort que d'autres également génériques.

\section{Généricité et morpho-sémantique}

\subsection{Généricité et propriétés}

Avant d'examiner quelques problèmes de morpho-sémantique, je ferai tout d'abord le lien entre certains types de propriété et la généricité. Reprenant et précisant des distinctions que j’ai fréquemment utilisées ${ }^{17}$, je distinguerai et opposerai :

a) Les propriétés essentielles et les propriétés accidentelles : on considère habituellement que $\mathrm{P}$ est une propriété essentielle de la classse $\mathrm{C}$ si elle est commune aux éléments de $\mathrm{C}$, et qu'elle est accidentelle si elle n'est partagée que par les éléments d'une sous-classe de C. De ce point de vue, «respirer l'oxygène de l'air» est une propriété essentielle de la classe des humains, et «faire de la pêche sous-marine » une propriété accidentelle de la même classe. Or cette "définition» est en fait problématique. Considérons en effet un chat quelconque : on ne s'étonnera pas qu'il ait quatre pattes, il s'agit donc d'une propriété intrinsèque essentielle de la classe des chats. Supposons que ce même chat perde une patte dans un accident : non seulement il ne perd pas son statut de chat, mais l'existence d'un tel chat à trois pattes ne remet pas en question le caractère constitutif que constitue la possession pour un chat de quatre pattes en bonne et due forme. On voit immédiatement que la définition de essentiel/accidentel passe par les phrases génériques, ce qui donne :

${ }^{17}$ Cf. par exemple [Anscombre, 1990], «Pourquoi un moulin à vent n'est pas un ventilateur », Langue française, 86, pp. 103-125 ; également [Anscombre, 1994]. 
(Déf) $\mathrm{P}$ est une propriété essentielle d'une classe $\mathrm{C}$ si Les $\times$ sont $\mathrm{P}$ est une phrase générique valide. $\mathrm{P}$ est une propriété accidentelle de $\mathrm{C}$ si Certains $x$ sont $P$ est générique valide, Les $x$ sont $P$ n'étant évidemment pas valide.

b) Les propriétés intrinsèques et les propriétés extrinsèques : est propriété intrinsèque d'une entité toute propriété qui en est constitutive. Une propriété qui n'est pas intrinsèque sera extrinsèque. C'est par exemple une propriété intrinsèque d'un livre qu'il ait des pages, mais c'est en revanche une propriété extrinsèque qu'un livre soit relié plein cuir ou ait une reliure en carton. Or il est clair qu'on ne remet pas en question la possession par une entité d'une propriété intrinsèque. C'est pourquoi on ne dira jamais des choses comme Max est maladif, mais l'est-il aujourd'bui ?. Dans le cas de classes ou de sous-classes, cette remarque nous mène à la définition de intrinsèque/extrinsèque suivante :

(Déf) $\mathrm{P}$ est une propriété intrinsèque d'une classe $\mathrm{C}$ si la phrase reliant $\mathrm{C}$ à $\mathrm{P}$ est typifiante a priori. Si cette phrase est typifiante locale, la propriété est extrinsèque.

Il y a donc une correspondance parfaite entre les propriétés et les phrases génériques: les propriétés intrinsèques essentielles sont représentées par des génériques extensives typifiantes a priori (Les voitures ont quatre roues), les intrinsèques accidentelles par des génériques partitives typifiantes a priori (Certains Français sont blonds), les extrinsèques essentielles par des génériques extensives typifiantes locales (Les chats sont affectueux), et les extrinsèques accidentelles par des génériques partitives typifiantes locales (Certains voitures ont six roues).

Nous allons maintenant traiter quelques problèmes de morpho-sémantique précisément par le biais des phrases génériques et des définitions ci-dessus.

\subsection{Généricité, propriétés, et préfixe négatif in- :}

La morphologie fait souvent figure de parent pauvre dans la plupart des grammaires. Ainsi la Grammaire française de [Togeby, 1985] - pourtant excellente sous bien des rapports - expédie en quelques lignes le problème de la formation et de l'existence d'adjectifs à préfixe négatif in-: "...Les deux préfixes négatifs in- et antis'ajoutent uniquement à des noms (substantifs ou adjectifs)...in- se joint surtout à des adjectifs...Il existe aussi des adjectifs simples qui admettent le préfixe in-: indigne, inégal,...» (p. 15). Or cette explication non seulement n'explique rien, mais est partiellement fausse. Il y a, en effet, certains «trous lexicaux» relatifs à cette préfixation négative en in-. Ainsi - ce qui pose les limites de la conception classique de la morphologie comme passage formel d'un mot à un autre - il y a une liste non négligeable de mots à préfixe négatif in- et pour lesquels cependant la contre-partie positive n'existe pas. Par exemple la série: (*colore, incolore), (*forme, informe), (*mense, immense), (*odore, inodore), (*sapide, insipide $)^{18}$. Notons que de tels adjectifs s'appliquent ou s'appliqueraient - à des objets. Or un objet n'est appréhendé comme tel qu'au travers de nos cinq sens, i.e. si et seulement si il possède une couleur, une dimension, une forme, une odeur, une saveur. En d'autres termes, les phrases condensées dans :

18 Sapide a autrefois existé avec le sens « qui a du goût », i.e. « dont on remarque le goût ». L'adjectif a survécu en chimie, mais avec un autre sens, dans la désignation les corps sapides. 
Jean-Claude Anscombre

Les objets ont une $($ couleur + forme + dimension + odeur + saveur $)$.

sont des phrases génériques extensives, et elles représentent des propriétés essentielles intrinsèques.Vu qu'il existe des objets sans forme précise, ou sans couleur, ce sont des phrases typifiantes a priori, qui admettent donc des exceptions. Or l'étude que nous avons menée des phrases génériques nous conduit à deux règles :

$\left.\mathbf{( R}_{\mathbf{1}}\right)$ On ne peut prédiquer d'une entité une propriété intrinsèque essentielle, i.e. qui vient d'une phrase générique extensive typifiante a priori, puisque de telles phrases ne permettent pas les SN spécifiques.

( $\left.\mathbf{R}_{2}\right)$ Dans le cas de phrases génériques typifiantes, on peut dire d'une entité qu'elle s'écarte de la norme de sa classe d'appartenance, qu'elle est une exception.

En d'autres termes, les phrases condensées en :

(62) Certains objets n'ont pas de (couleur + dimension + forme + odeur + saveur $)$.

sont des génériques partitives typifiantes locales. Elles définissent dans le monde des objets des propriétés accidentelles, que rien n'empêche, on l'a vu, de prédire d'entités particulières : les génériques partitives admettent les $\mathrm{SN}$ spécifiques. Concluons : $\left(\mathrm{R}_{1}\right)$ fait qu'un objet ne peut être ni colore, ni forme, ni mense, ni odeur, ni sapide, mais $\left(\mathrm{R}_{2}\right)$ autorise une objet à être exceptionnel, anormal, et à être donc incolore, informe, imense, inodore, ou insipide.

Une fois ces règles mises en évidence, on s'aperçoit qu'elles permettent d'expliquer d'autres cas non moins insolites, ainsi par exemple des doublets atypiques comme (taché, *intachê) versus (maculé, immaculè). Supposons que intaché existe: il signifierait alors le contraire de taché, i.e. «qui n'est pas souillé par des taches ». Mais nous avons des choses une conception que l'on pourrait qualifier de virginale : si nous parlons de maisons en général ou de voitures, il s'agit naturellement de maisons en bon état et de voitures intactes ${ }^{19}$. C'est l'idée banale de la pureté originelle. En bref, la phrase générique :

$$
\text { (63) Les choses sont intachées (= " pures »). }
$$

non seulement est valide (et extensive), mais dénote une propriété intrinsèque, et est donc typifiante a priori. Elle n'autorise donc pas l'usage de intaché puisqu'elle n'admet pas les $\mathrm{SN}$ spécifiques (règle $\left.\left(\mathrm{R}_{1}\right)\right)$. On ne dit pas intaché parce qu'il s'agit là de l'état normal des choses de ce monde, au yeux de la langue du moins ${ }^{20}$. Comment se fait-il alors que l'on ait immaculé? En effet, maculé est très proche par le sens de taché, et l'application de $\left(\mathrm{R}_{1}\right)$, qui interdisait intaché devrait de la même façon empêcher immaculé. En fait, immaculé ne signifie pas exactement "sans tache », mais plutôt « sans absolument aucune tache ». Ce n'est donc pas $\left(\mathrm{R}_{1}\right)$ qui s'applique au travers de la

${ }^{19}$ Le dogme chrétien du péché originel s'oppose précisément à cette conception des choses, du moins dans le domaine métaphysique.

${ }^{20}$ D'où intact = «qui n'a pas été touché », qui permet de rendre l'idée de intaché tout en évitant le paradoxe. Ce n'est pas le seul cas de ce type. 
générique extensive Les choses sont pures, mais $\left(\mathrm{R}_{2}\right)$ via cette fois la générique partitive Certaines choses sont plus pures que d'autres ${ }^{21}$ : laquelle admet bien sûr, les $\mathrm{SN}$ spécifiques.

\subsection{Généricité, propriétés, et noms d'agent en-eur}

Les grammaires ne voient habituellement pas de problème particulier dans la formation des noms d'agent en -eur. Ainsi, Grevisse affirme du suffixe -eur qu'il «...s'ajoute aux verbes et aux noms et donne maintenant des noms d'agent... » (Le bon usage, 1980, p. 104). On remarque immédiatement que, en se bornant aux verbes, le dérivé n'a de chances d'exister que si le verbe est agentif ${ }^{22}$. On n'a ni alleur, ni veneur, ni boiteur, ni tombeur, et encore moins frissonneur ou transpireur. Ce qui ne nous tire pas d'affaire pour autant. Il est usuel de définir un $V$-eur comme quelqu'un «qui a la propriété de $V$-er». Cette définition s'applique certes dans un nombre important de cas, ainsi : travailleur à mi-temps = "qui travaille à mi-temps ", nageur = "qui nage », prêteur sur gages $=$ « qui prête sur gages »,...etc. Mais beaucoup d'exemples s'écartent de cette norme : soit le dérivé n'existe pas seul (*arleur/beau parleur), soit le sens n'est pas celui qui est attendu. Ainsi voyeur, qui ne signifie jamais «qui voit», ou faiseur, dont le sens n'est pas «qui fait ». C'est à quelques uns de ces cas que je vais me consacrer à présent. Un premier cas sera l'impossibilité d'énoncés comme Mon fils est un mangeur alors qu'on a très bien en revanche Mon fils est un gros mangeur. A la lumière du paragraphe précédent, on voit ce qui se passe. L'énoncé :

\section{(64) Les hommes mangent.}

avec manger = «ingérer des aliments », est une phrase générique extensive typifiante a priori, dénotant une propriété intrinsèque essentielle. Il est dans la nature même du genre humain qu'il ait à se nourrir. A ce titre, et selon $\left(\mathrm{R}_{1}\right)$, elle n'admet pas les $\mathrm{SN}$ spécifiques. On ne peut dire Mon fils mange, et donc Mon fils est mangeur - qui en est la transposition directe 23 - est également exclu. En revanche, des phrases comme :

(65) Certains hommes mangent beacoup.

(66) Certains hommes mangent (des frites + des grenouilles + de la choucroute).

sont également génériques mais cette fois partitives, les extensives correspondantes n'étant pas valides. Elles admettent les SN spécifiques, à savoir gros mangeur et mangeur de (frites + grenouilles + choucroute). On a un phénomène analogue pour buveur. Boire est en effet ambigu entre boire 1 = «ingérer des liquides », et boire 2 = «ingérer de l'alcool ». L'homme devant boire 1 par nature, buveur ne signifiera donc jamais, en vertu de $\left(\mathrm{R}_{1}\right)$, "qui ingère des liquides». On aura en revanche buveur (d'eau + de lait), selon $\left(\mathrm{R}_{2}\right)$, puisque cette spécialisation de boire 1 correspond à des phrases génériques partitives. De la même façon, on aura un (gros) buveur = «qui boit2 (beaucoup) », puisqu’à boire 2 correspond la phrase partitive Certains bommes boivent 2 (beaucoup).

Généralisons : un nom d'agent de type $V$-eur correspond :

\footnotetext{
${ }^{21}$ Remarquons que ce principe de l'écart de la norme par excès est celui qui est utilisé dans certaines publicités. Ainsi pour définir des lessives qui lavent plus blanc que blanc!

${ }^{22} C f$. sur ce sujet les travaux de A. Zribi Hertz sur les verbes ergatifs.

${ }^{23}$ Sur la notion de transposition, of. [Benveniste, 1974, pp. 113-125].
} 
Jean-Claude Anscombre

a) A une interprétation agentive du verbe $V$ de base.

b) A une interprétation de $V$ issue d'une phrase générique partitive, i.e. renvoyant à une propriété accidentelle.

Lorsque ces deux propriétés ne sont pas remplies, le nom d'agent n'existe pas. On comprend alors pourquoi un voyeur ne sera jamais quelqu'un qui possède la vue, mais nécessairement une personne qui est agent de sa vision, donc qui regarde; et qui regarde certaines choses que d'autres ne regardent pas. Soit maintenant le cas de pleurer. Dans son sens normal, il s'agit d'un verbe ergatif, et le nom d'agent n'existe pas. Une phrase comme Ce bébé est un gros pleureur est étrange. Mais on a bel et bien pleureuse pour désigner la profession (caractère agentif et intrinsèque) peu courante (phrase générique partitive) qui consiste à être rémunéré pour pleurer dans les enterrements. Dernier point : j'ai dit que ces noms d'agent désignaient des propriétés accidentelles. Or, pour reprendre une remarque antérieure, ces propriétés peuvent être aussi bien intrinsèques qu'extrinsèques. Ainsi danseur désigne aussi bien l'aptitude à danser (propriété intrinsèque) - d'où son utilisation pour désigner la profession - que celui qui danse occasionnellement (propriété extrinsèque).

Jean-Claude ANSCOMBRE (LLI)

MSH (Bureau 234B)

54 Boulevard Raspail

F 75270 Paris Cédex 06

anscombr@lli.univ-paris13.fr 


\section{BIBLIOGRAPHIE}

ANSCOMBRE (J.C.)

1990, «Les syllogismes en langue naturelle. Déduction logique ou inférence discursive ? ", Cabiers de linguistique française, 11, pp. 215-240.

1994, «L'insupportable légèreté morphologique du préfixe négatif in-dans la formation d'adjectifs », Actes du colloque «La Négation », Linx, numéro spécial, Paris X-Nanterre, pp. 299-321.

1995, La théorie des topoï, Kimé, París.

1996, «Semántica y léxico : topoi, estereotipos y frases genéricas », Revista española de lingüistica, 25, 2, pp. 297-310.

1995-1996 (paru 1997), «La semántica y las frases genéricas : viejos problemas y nuevos enfoques », Cuadernos de filología francesa, 9, pp. 7-22.

1999, «Le jeu de la prédication dans certains composés nominaux », Langue française, 122 , pp. 52-69.

2001, «Les syntagmes les N/des N en position objet : un syntagme générique ou pas ? , à paraître in Linguisticae Investigationes.

2001, « Sens, référence et contexte, dans la théorie des stéréotypes », Langages, à paraittre.

2001, «A propos du critère de gnomicité/épisodicité de K. Carlson », à paraitre dans Mélanges Jonasson, Uppsala (Suède), août 2001.

2001, «A propos des mécanismes sémantiques de formation de certains noms d'agent en français et en espagnol », Langages, 143, pp. 28-48.

1961, Philosophical Papers, Clarendon Press, Oxford.

BENVENISTE (E.), 1974, Problèmes de linguistique générale, II, Ed. Gallimard, Paris.

CARLSON (G.N.), 1982, « Generic Terms and Generic Sentences », Journal of Philosophical Logic, 11, pp. $145-181$.

DAHL (Ö.), 1985, « Remarques sur le générique », Langages, 85, pp. 55-60.

DUCROT (O.), 1970, «Les indéfinis et l'énonciation », Langages, 5, n¹7, pp. 91-111.

GALMICHE (M.), 1985, «Phrases, syntagmes, et articles génériques », Langages, 85, pp. 2-39.

1986, "Référence indéfinie, événements, propriétés et pertinence », in J. David et G.Kleiber, Déterminants : syntaxe et sémantique, Recherches linguistiques, XI, pp. 41-71, Paris, Klincksieck.

GEACH (P. T.), 1967, Reference and Generality, Cornell University Press.

GUERICOLAS (C.), 1987, «Les phrases dispositionnelles. Une approche informelle », in Rencontres avec la généricité, G. Kleiber éd., Recherches linguistiques, XII, pp. 33-56.

JAKENDOFF (R.), 1983, Semantics and Cognition, MIT Press, Cambridge (Mass.). 
Jean-Claude Anscombre

JESPERSEN (O.), 1971, La philosophie de la grammaire, Minuit, Paris.

$\operatorname{KLEIBER}(\mathrm{G}$.

1978, "Phrases et valeurs de vérité », in La notion de recevabilité en linguistique, ed. R.Martin, Klincksieck, París, pp. 21-65.

1983, « Remarques sur la généricité et la spécificité », Le français moderne, 51, n¹, pp. 3649.

1987, Du côté de la référence verbale : les phrases habituelles, Peter Lange, Berne.

1988, «Phrases génériques et raisonnement par défaut », Le français moderne, 56, n¹/2, pp 1-15.

KURODA (S.Y.), 1973, «The Categorical and the Thetic Judgments : Evidence from Japanese Syntax », Foundations of Language, 9.2, pp. 153-185.

MC CAWLEY (James D.), 1981, Everything that Linguists have Always Wanted to Know about Logic, The University of Chicago Press, Chicago.

MARTIN (R.), 1985, « Aspects de la phrase analytique », Langages, 85, pp. 40-54.

PUTNAM (H.), 1975, "The meaning of 'meaning' », Philosophical Papers, vol. 2, Cambridge University Press, pp. 215-271.

1975, «Analicity and apriority : beyond Wittgenstein and Quine », Philosophical Papers, vol.3, pp. 115-138.

QUINE (W. van O.)

1963, From a Logical Point of View, Harper and Row, New-York.

1967, «The A priori and the Analytic », in Philosophical Logic, ed. P. F.Strawson, Oxford University Press, pp. 107-128, London.

STRAWSON (P. F.), 1971, Études de logique et de linguistique, Seuil, Paris. 